Journal of the Operations Research

Society of Japan

2003, Vol. 46, No. 4, 467-486

\title{
THE CENTRALIZED SEQUENTIAL INVESTMENT PROBLEM WITH REGIONAL CHARACTERISTICS EFFECT BY USING GENETIC ALGORITHMS
}

\author{
Cheng-Chang Chang \\ National Defense University
}

(Received May 5, 2002; Revised June 9, 2003)

\begin{abstract}
This research aims to find a non-recurring directed path that will enable all investment regions to achieve the target revenues within the shortest period of time. We call this optimization problem the centralized sequential investment problem (CSIP). "Regional characteristics effect (RC effect)" is assumed to be the major factor that affects the expected time to achieve the target revenue for an investment region. Using the RC effect definition, we introduce the concepts of path effectiveness and sequential effectiveness. Furthermore, we define a completely memoryless property of the $\mathrm{RC}$ effect and consider the scenario that the $\mathrm{RC}$ effect of a prior investment region on a subsequent investment region follows a linear fashion. Accordingly, this paper explores CSIP's structural properties and constructs a binary integer programming model for transnational sequential investment. Also, a simple method of estimating model parameters and a GA-based solution procedure are proposed for solving CSIP.
\end{abstract}

Keywords: Centralized sequential investment, regional characteristic effect, path effectiveness, sequential effectiveness, genetic algorithms

\section{The Centralized Sequential Investment Strategy}

In today's knowledge-based economy where competency plays a more critical role than ever in corporate survival and growth, companies are all committed to ensuring their sustained competitive advantages and globalising their operations. In this paper, we propose an alternative model for transnational investment under the centralized sequential investment strategy. We consider a company that plans to select $N$ international regions for transnational marketing and sales of some end products or services. We will suppose that the firm wishes to use the market entry mode of foreign direct investment, such as sole ownership and acquisition of whole ownership. (Related extensions based on international entry models can be found in $[2,3,5])$. Based on the above assumptions, the optimization of a centralized sequential investment problem (CSIP) aims to find a non-cyclic path minimizing the expected time to achieve target revenue for every investment region. To construct the CSIP, the centralized sequential investment strategy must be described first. Let $P$ be the non-cyclic directed path that starts at the parent company, $P \in \Omega_{N}$, where $\Omega_{N}$ is the set of all non-recurring directed paths in between $N$ planned investment regions. Also, let $P_{i}$ be $i$ th investment region of path $P, \forall P \in \Omega_{N}$ and $T_{P_{i} \mid P}$ be the time to achieve target revenue $R_{P_{i}}$ for investment region $P_{i}, i=0,1,2, \ldots, N$ ( $P_{0} \equiv 0$ representing the parent company), considering the influence of regional characteristics on path $P$ with the limited amount of capital $C_{0}$ initially invested by the company. Based on the notations and definitions above, the centralized sequential investment strategy is illustrated in Figure 1 as follows: 


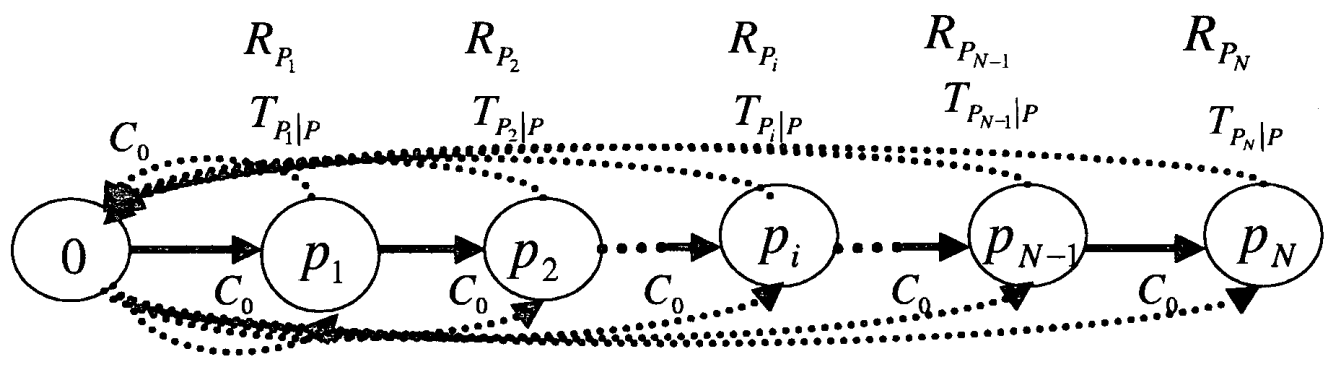

Figure 1: Centralized sequential investment strategy (Number "0" represents parent company, the dotted line represents the direction of capital flow, and the solid line represents the transnational investment sequence.)

As shown in the above diagram, under the centralized sequential investment strategy, the parent company concentrates its capital $C_{0}$ (after the parent company achieves target revenue $R_{0}$ ) in one investment region and will not transfer the initial capital investment to another region until the prior region achieves the target revenue. The investment continues by such a sequence and gradually grows transnational until investment in all planned regions is completed and has gained a return (the target revenue). When an investment region achieves its target revenue, we say this investment region has gleaned the sufficient cash flow to respond to any operational risk and thus can maintain its operation as stably as possible.

\section{The CSIP's Initial Formulation}

With the limited human, material, and financial resources available, considering the on-going global recession, companies that intend to expand their initial operational scale usually reduce risks involved in doing so by centralizing their resources through the centralized sequential investment strategy. According to the definition of the centralized sequential investment strategy proposed in Section 1, the CSIP refers to transnational corporations pursuing an optimal non-recurring directed path that enables corporations to achieve the target revenues for all investment regions within the shortest expected time period. In other words, if we let $P^{*}$ be the optimal sequential investment path, then

$$
P^{*}=\arg \min _{P \in \Omega_{N}}\left\{E\left[T_{P_{1} \mid P}+T_{P_{2} \mid P}+\cdots+T_{P_{N-1} \mid P}+T_{P_{N} \mid P}\right]\right\}
$$

The expected time to achieve the target revenue for an investment region is extremely hard to estimate since it also depends on the prior invested regions. It is both theoretically and practically difficult to obtain $P^{*}$ merely based on the above model. This study will devote the following sections to first discussing the factors (regional characteristics) that affect the expected time to achieve target revenues for all investment regions. Next we introduce the concepts of path effectiveness and sequential effectiveness and convert the original problem of "minimizing the expected time to achieve the target revenues for all investment regions" into the problem of "maximizing the total of all regions' weighted sequential effectiveness". By doing so, we can estimate the sequential effectiveness directly instead of estimating the expected time to achieve target revenue for an investment region, after which we can find the optimal sequential investment path. 


\section{Related Terminology}

\subsection{Regional characteristics effect (RC effect)}

Under centralized sequential investment strategy, if a prior investment region's consumer environment and consumer culture possesses a certain consumer fervor, this will have an effect on the consumer behaviors regarding a certain commodity or service in subsequent investment regions. When this happens, we say that the regional characteristics (RC) effect arises within the investment sequence. For a specified prior investment region, the higher demand for this commodity or service in this region implies that there has been a larger RC effect on the subsequent investment regions.

\subsection{Consumer fervor index (CFI)}

Consider the influence of the regional characteristics effect on path $P, P \in \Omega_{N}$. Assume region $P_{i}$ 's regional characteristics effect on subsequent investment regions is in the form of demand when the target revenue $\left(R_{P_{i}}\right)$ achieved can be quantified by an ordinal scale, $\lambda_{P_{i} \mid P}$. The variable $\lambda_{P_{i} \mid P}$ is called the "consumer fervor index (CFI)". The larger value of $\lambda_{P_{i} \mid P}$ reflects the higher RC effect (in terms of consumer fervor) that region $P_{i}$ has on subsequent investment regions. In addition, let $\lambda_{P_{i} \mid P}=\lambda_{P_{i}}+\Delta \lambda_{P_{i} \mid P}$, where $\lambda_{P_{i}}$ is any positive real number representing the CFI when region $P_{i}$ achieves target revenue $R_{P_{i}}$ regardless of the prior investment regions' RC effect. Moreover, $\Delta \lambda_{P_{i} \mid P}\left(\Delta \lambda_{P_{i} \mid P}>0\right)$ represents the increased amount of CFI when region $P_{i}$ achieves target revenue $R_{P_{i}}$ if the prior investment regions' $\mathrm{RC}$ effect on region $P_{i}$ is considered. Since the CFI, $\lambda_{P_{i} \mid P}$, is defined as an ordinal scale, we can assume that $E\left[T_{P_{i} \mid P}\right] \rightarrow 0$ when $\lambda_{P_{i} \mid P}=2 \lambda_{P_{i}}$. Since the fact that $E\left[T_{P_{i} \mid P}\right]$ is extremely short represents the extremely great demand in region $P_{i}$ at that time that target revenue $R_{P_{i}}$ is achieved. As a result, we can assume that $\operatorname{Max}_{P \in \Omega_{N}}\left\{\lambda_{P_{i} \mid P}\right\} \leq 2 \lambda_{P_{i}}$. Due to the fact that $\lambda_{P_{i} \mid P}=\lambda_{P_{i}}+\Delta \lambda_{P_{i} \mid P}$, we can obtain that $0<\Delta \lambda_{P_{i} \mid P} \leq \lambda_{P_{i}}$ for any path $P, P \in \Omega_{N}$.

\subsection{Regional characteristics correlation index (RCCI)}

Let $\lambda_{P_{i-k}, P_{i}}$ be the CFI when region $P_{i}$, under the influence of the regional characteristics of region $P_{i-k}$, achieves the target revenue and the CFI in region $P_{i-k}$ achieves $\lambda_{P_{i-k}}, k=$ $1,2, \ldots, i ; i=1,2, \ldots, N$. Also, $\lambda_{P_{i-k}, P_{i}}^{u}$ denotes the CFI when region $P_{i}$, considering the RC effect from region $P_{i-k}$, achieves the target revenue and the CFI in region $P_{i-k}$ is $2 \lambda_{P_{i-k}}$. $\lambda_{P_{i-k}, P_{i} \mid P}$ represents the CFI when region $P_{i}$, considering the $\mathrm{RC}$ effect from region $P_{i-k}$, achieves the target revenue and the CFI in region $P_{i-k}$ is $\lambda_{P_{i-k} \mid P}$. In this paper we assume that there exists a real number $\theta_{P_{i-k}, P_{i}}$ so that

$$
\frac{\lambda_{P_{i-k}, P_{i} \mid P}-\lambda_{P_{i-k}, P_{i}}}{\lambda_{P_{i-k}, P_{i}}^{u}-\lambda_{P_{i-k}, P_{i}}} \approx\left(\frac{\lambda_{P_{i-k} \mid P}-\lambda_{P_{i-k}}}{2 \lambda_{P_{i-k}}-\lambda_{P_{i-k}}}\right)^{\theta_{P_{i-k}, P_{i}}} .
$$

We call this "regional characteristics correlation index (RCCI)".

If $\theta_{P_{i-k}, P_{i}}<1$, the consumers in region $P_{i}$ that are affected by region $P_{i-k}$ consumers will fervently follow the behavior of the consumers in region $P_{i-k}$. The consumers in region $P_{i}$ that are affected by region $P_{i-k}$ consumers will be disinclined to follow the behavior of the consumers in region $P_{i-k}$, if $\theta_{P_{i-k}, P_{i}}>1$. If $\theta_{P_{i-k}, P_{i}}=1$, then the consumers in region $P_{i}$ are neither hot nor cool (fervent nor disinclined) about following the behavior of the consumers in region $P_{i-k}$. (Note that $\theta_{P_{i-k}, P_{i}}$ may be not equal to $\theta_{P_{i}, P_{i-k}}$ ). In this paper we use both indices CFI and RCCI to measure the magnitude of RC effect that a prior investment region has on a subsequent investment region. 


\subsection{Path effectiveness}

Path effectiveness refers to the percentage of reduction of the expected time to achieve the target revenues for all investment regions that can be attributed to the consideration of $\mathrm{RC}$ effect. In other words, consider an investment path $P, P \in \Omega_{N}$. Let $U(P)$ represent path effectiveness, then

$$
U(P)=\frac{E\left[\sum_{i=1}^{N} T_{P_{i}}\right]-E\left[\sum_{i=1}^{N} T_{P_{i} \mid P}\right]}{E\left[\sum_{i=1}^{N} T_{P_{i}}\right]} .
$$

The random variable $T_{P_{i}}$ is the time to achieve the target revenue $\left(R_{P_{i}}\right)$ when we do not take into account the $\mathrm{RC}$ effect on path $P$.

\subsection{Sequential effectiveness}

Sequential effectiveness refers to the percentage of reduction of the expected time to achieve the target operation revenue for an investment region that results from $\mathrm{RC}$ effect on the prior investment regions. In other words, if we let $F_{P_{i} \mid P}$ be region $P_{i}$ 's sequential effectiveness in path, $P, P \in \Omega_{N}$, then

$$
F_{P_{i} \mid P}=\frac{E\left[T_{P_{i}}\right]-E\left[T_{P_{i} \mid P}\right]}{E\left[T_{P_{i}}\right]}
$$

\subsection{Pure and multi-dimension-based sequential effectiveness}

If we suppose that $\lambda_{P_{i-1} \mid P}, \lambda_{P_{i-2} \mid P}, \ldots, \lambda_{P_{0} \mid P}$ influence $F_{P_{i} \mid P}$ independently and let $f_{\left(P_{i-k}, P_{i}\right) \mid P}$ be $\lambda_{P_{i-k} \mid P}$ 's strictly increasing function, then

$$
F_{P_{i} \mid P}=\sum_{k=1}^{i} f_{\left(P_{i-k}, P_{i}\right) \mid P}\left(\lambda_{P_{i-k} \mid P}\right)
$$

In addition, let $f_{\left(P_{i-k}, P_{i}\right) \mid P}=h_{\left(P_{i-k}, P_{i}\right) \mid P}+g_{\left(P_{i-k}, P_{i}\right) \mid P}$, where $h_{\left(P_{i-k}, P_{i}\right) \mid P}$ is a function of $\lambda_{P_{i-k}}$, and $g_{\left(P_{i-k}, P_{i}\right) \mid P}$ is a function of $\Delta \lambda_{P_{i-k} \mid P}$ and $\lambda_{P_{i-k}}$. We say $h_{\left(P_{i-k}, P_{i}\right) \mid P}$ belongs to the class of pure sequential effectiveness and $g_{\left(P_{i-k}, P_{i}\right) \mid P}$ belongs to the class of multi-dimensionbased sequential effectiveness. Since the value of $\Delta \lambda_{P_{i-k} \mid P}$ depends on $\lambda_{P_{i-k-l} \mid P}, l=$ $1,2, \ldots, i-k-1$, hence, we further suppose $g_{\left(P_{i-k}, P_{i}\right) \mid P} \approx \sum_{j=1}^{i-k-1} g_{\left(P_{i-k}, P_{i}\right) \mid P}^{j}$ where $g_{\left(P_{i-k}, P_{i}\right) \mid P}^{j}$ is termed $(j+1)$-dimension-based sequential effectiveness, because the value of $g_{\left(P_{i-k}, P_{i}\right) \mid P}^{j}$ depends on $(j+1)$ number of simple CFIs (for convenience, we term $\lambda_{P_{i} \mid P}$ the complex CFI and term $\lambda_{P_{i}}$ the simple CFI). According to the definition of multi-dimension-based sequential effectiveness, we suppose that $g_{\left(P_{i-k}, P_{i}\right) \mid P}^{j}$ is strictly decreasing in $j$.

An example will illustrate the difference between pure and multi-dimension-based sequential effectiveness. Assume a company plans to invest in the three regions A, B, and C. The investment starts with region $\mathrm{A}$, moves on to region $\mathrm{B}$ after region $\mathrm{A}$ achieves target revenue, and ends with region $C$. Since the commodity or service is in great demand or is popular (because target revenue has been achieved) in region $\mathrm{A}$, an additional demand can be created in region $B$ as a result of region A's regional characteristics effect on region B. In other words, the consumer behavior in region $B$ is prompted by the fact that this commodity or service is in great demand or is popular in region $\mathrm{A}$. The addition of this demand reduces the time to achieve the target revenue for region $\mathrm{B}$. The percentage of reduction of the expected time is the sequential effectiveness produced by region A's RC effect on region B. Obviously this sequential effectiveness is only determined by region A's 
simple CFI, thus it is called pure sequential effectiveness. By the same token, the consumer behavior in region $\mathrm{C}$ is prompted by region B's $\mathrm{RC}$ effect. Region A's $\mathrm{RC}$ effect increases the demand in region $\mathrm{B}$ when target revenue is achieved, which in turn increases sequential effectiveness of region $\mathrm{C}$ attributed to region $\mathrm{B}$. Since this increased sequential effectiveness is related to both region A and region B's simple CFIs, it is called two-dimension-based sequential effectiveness.

\section{Model Construction and Property Discussion}

Theorem $1 \operatorname{Min}_{P \in \Omega_{N}}\left\{E\left[\sum_{i=1}^{N} T_{P_{i} \mid P}\right]\right\} \cong \operatorname{Max}_{P \in \Omega_{N}}\{U(P)\}$.

Proof:

$$
\begin{aligned}
\operatorname{Min}_{P \in \Omega_{N}}\left\{E\left[\sum_{i=1}^{N} T_{P_{i} \mid P}\right]\right\} & \cong \operatorname{Max}_{P \in \Omega_{N}}\left\{-E\left[\sum_{i=1}^{N} T_{P_{i} \mid P}\right]\right\} \cong \operatorname{Max}_{P \in \Omega_{N}}\left\{E\left[\sum_{i=1}^{N} T_{P_{i}}\right]-E\left[\sum_{i=1}^{N} T_{P_{i} \mid P}\right]\right\} \\
& \cong \operatorname{Max}_{P \in \Omega_{N}}\left\{\frac{E\left[\sum_{i=1}^{N} T_{P_{i}}\right]-E\left[\sum_{i=1}^{N} T_{P_{i} \mid P}\right]}{E\left[\sum_{i=1}^{N} T_{P_{i}}\right]}\right\}=\operatorname{Max}_{P \in \Omega_{N}}\{U(P)\} .
\end{aligned}
$$

(Note: For two optimization problems $\mathrm{A}$ and $\mathrm{B}$, "Problem $\mathrm{A} \cong$ Problem B" means the optimal feasible solution of Problem A is the same as the solution of Problem B; on the other hand, "Problem A = Problem B" means that not only the optimal feasible solution to Problem A but also the optimal value of objective function of Problem $A$ is the same as Problem B.)

Remark 1: Theorem 1 shows that minimization of "the expected time to achieve the target revenues for all investment regions" can be converted to maximization of "path effectiveness". Since path effectiveness is the percentage of reduction of the expected time for accomplishing the overall investment goal as a result of the effect that investment sequence has on investment performance, the conversion of path effectiveness helps explain the differentials in investment performance among various feasible investment paths.

Since $E\left[T_{P_{i}}\right]$ may vary, let $\alpha_{P_{i}}$ be the relative weight of $E\left[T_{P_{i}}\right]$, where there exists a random variable $T$ that makes $E\left[T_{P_{i}}\right]=\alpha_{P_{i}} E[T]$ (Note that $\sum_{i=1}^{N} \alpha_{P_{i}}=1$ ). The relative weight of $E\left[T_{P_{i}}\right]$ can be estimated directly (for example, use AHP pair comparison, see $[12])$.

Theorem $2 \quad \operatorname{Max}_{P \in \Omega_{N}} U(P)=\operatorname{Max}_{P \in \Omega_{N}}\left\{\sum_{i=1}^{N} \alpha_{P_{i}} F_{P_{i} \mid P}\right\}$.

Proof: The result of Theorem 1 can be obtained by the inference below.

$$
\operatorname{Max}_{P \in \Omega_{N}} U(P)=\operatorname{Max}_{P \in \Omega_{N}}\left\{\frac{E\left[\sum_{i=1}^{N} T_{P_{i}}\right]-E\left[\sum_{i=1}^{N} T_{P_{i} \mid P}\right]}{E\left[\sum_{i=1}^{N} T_{P_{i}}\right]}\right\}
$$




$$
\begin{aligned}
& =\operatorname{Max}_{P \in \Omega_{N}}\left\{\frac{\sum_{i=1}^{N}\left(\alpha_{P_{i}} E[T]-E\left[T_{P_{i} \mid P}\right]\right)}{\sum_{i=1}^{N} \alpha_{P_{i}} E[T]}\right\}=\operatorname{Max}_{P \in \Omega_{N}}\left\{\frac{\sum_{i=1}^{N}\left(\alpha_{P_{i}} E[T]-E\left[T_{P_{i} \mid P}\right]\right)}{E[T]}\right\} \\
& =\operatorname{Max}_{P \in \Omega_{N}}\left\{\sum_{i=1}^{N}\left(\frac{\alpha_{P_{i}} E[T]-E\left[T_{P_{i} \mid P}\right]}{E[T]}\right)\right\}=\operatorname{Max}_{P \in \Omega_{N}}\left\{\sum_{i=1}^{N}\left(\alpha_{P_{i}} \frac{\alpha_{P_{i}} E[T]-E\left[T_{P_{i} \mid P}\right]}{\alpha_{P_{i}} E[T]}\right)\right\} \\
& =\operatorname{Max}_{P \in \Omega_{N}}\left\{\sum_{i=1}^{N} \alpha_{P_{i}} \frac{E\left[T_{P_{i}}\right]-E\left[T_{P_{i} \mid P}\right]}{E\left[T_{P_{i}}\right]}\right\}=\operatorname{Max}_{P \in \Omega_{N}}\left\{\sum_{i=1}^{N} \alpha_{P_{i}} F_{P_{i} \mid P}\right\} .
\end{aligned}
$$

Remark 2: The result of Theorem 2 means that minimization of "the expected time required for every investment region to glean the target revenue" can be converted to maximization of "the total of all regions' weighted sequential effectiveness".

For the sake of convenience, we use the numbers $1,2, \ldots, N$ to label the investment regions, that is, $S=\{1,2, \ldots, N\}$, where $S$ denotes the set of planned investment regions. For further study of the model's properties, the relevant symbols are defined as follows:

$W_{i j}^{R}=$ A greatest lower bound (infimum) of $f_{(i, j) \mid P}\left(\lambda_{i \mid P}\right) ; j \in S \cup\{0\} ; j \in S \cup\{0\} ; j \in$ $S ; i \neq j$. Since $\lambda_{i} \leq \lambda_{i \mid P} \leq 2 \lambda_{i}, W_{i j}^{R}$ refers to region $j$ 's (pure) sequential effectiveness that can be attributed to region $i$ 's consumer fervor characteristics effect shown in index $\lambda_{i}$.

$U_{i j}^{R}=$ A least upper bound (supremum) of $f_{(i, j) \mid P}\left(\lambda_{i \mid P}\right), \forall i, j \in S ; i \neq j$. In other words, $U_{i j}^{R}$ refers to region $j$ 's sequential effectiveness that can be attributed to region $i$ 's consumer fervor shown in index $2 \lambda_{i}$.

$W_{(i j) k}^{R}=$ Region $k$ 's two-dimension-based sequential effectiveness that can be attributed to region $i$ and $j$ 's consumer fervors shown in index $\lambda_{i}$ and $\lambda_{j}$. In other words, region $i$ 's consumer fervor shown in index $\lambda_{i}$ increases the demand when region $j$ achieves the target revenue $\left(R_{j}\right)$, which will in turn intensify region $j$ 's regional characteristics effect on region $k$. The intensified regional characteristics effect that region $j$ has on region $k$ depends on two CFIs, $\lambda_{i}$ and $\lambda_{j}$, and it contributes to a two-dimension-based sequential effectiveness, $W_{(i j) k}^{R}, \forall i \in S \cup\{0\} ; j, k \in S ; i \neq j \neq k$.

The above notations related to sequential effectiveness can be illustrated by the following investment path chart for the three investment regions $i, j$, and $k$ (see Figure 2). That is, Figure 2 shows that the different types of sequential effectiveness among regions $i, j$, and $k$.

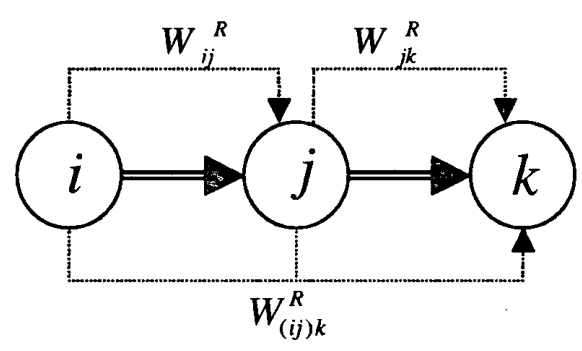

Figure 2: Sequential effectiveness chart 
Since $\lambda_{j \mid P}$ refers to the consumer fervor in region $j$ when the target revenue $\left(R_{j}\right)$ is achieved, and $\lambda_{j \mid P}$ is ranged on $\left[\lambda_{j}, 2 \lambda_{j}\right]$, we suppose there exists a real number $\phi_{j} \leq 1$ such that

$$
F_{j \mid P}=\frac{E\left[T_{j}\right]-E\left[T_{j \mid P}\right]}{E\left[T_{j}\right]} \approx\left(\frac{\lambda_{j \mid P}-\lambda_{j}}{\lambda_{j}}\right)^{\phi_{j}}, \quad \forall j \in S .
$$

Here $E\left[T_{j \mid P}\right]$ is a strictly decreasing convex function of $\lambda_{j \mid P}$ in the interval $\left[\lambda_{j}, 2 \lambda_{j}\right]$ in the sense that the more agressive the consumer behavior in region $j$ the smaller $\phi_{j}$ will be; i.e., the consumers will increase the demand rate significantly even if only some specified people buy the product. When $\phi_{j}$ approaches the value 1 , the consumption behavior in region $j$ is very conservative for the product and the demand rate only increases significantly when a great deal of people buy the product. As this paper represents the introduction of a new theory, for the sake of model simplicity we only initially consider the case that $\phi_{j}$ approaches the value 1 . This case $\left(\phi_{j}\right.$ approaches the value 1$)$ can be illustrated in Figure 3 as follows:

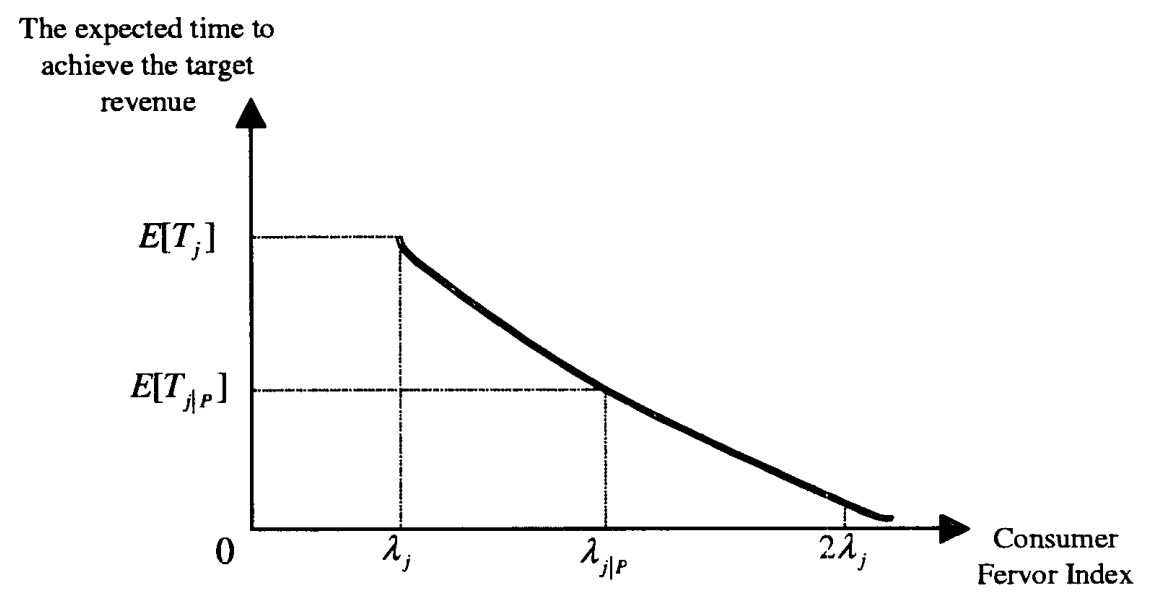

Figure 3: A negative linear relationship between the consumer fervor index and the expected time

As a result, Equation (6) can be rewritten as follows:

$$
F_{j \mid P}=\frac{\lambda_{j \mid P}-\lambda_{j}}{\lambda_{j}}, \quad \forall j \in S
$$

Since $\lambda_{P_{i-1} \mid P}, \lambda_{P_{i-2} \mid P}, \ldots, \lambda_{P_{0} \mid P}$ influence $F_{P_{i} \mid P}$ independently (see Section 3.6), we know that

$$
F_{P_{i} \mid P}=\frac{\Delta \lambda_{P_{i} \mid P}}{\lambda_{P_{i}}}=\frac{\sum_{k=1}^{i}\left(\lambda_{P_{i-k}, P_{i} \mid P}-\lambda_{P_{i}}\right)}{\lambda_{P_{i}}} .
$$

According to Equations (5) and (7), we can obtain that

$$
f_{\left(P_{i-k}, P_{i}\right) \mid P}\left(\lambda_{P_{i-k} \mid P}\right)=\frac{\lambda_{P_{i-k}, P_{i} \mid P}-\lambda_{P_{i}}}{\lambda_{P_{i}}} .
$$

According to Equation (9) and definition of $W_{i j}^{R}$ and $U_{i j}^{R}$, we can obtain Equations (10) and (11) as follows:

$$
W_{i j}^{R}=\inf \left\{f_{(i, j) \mid P}\left(\lambda_{i \mid P}\right): \lambda_{i} \leq \lambda_{i \mid P} \leq 2 \lambda_{i}\right\}
$$




$$
=f_{(i, j) \mid P}\left(\lambda_{i}\right)=\frac{\lambda_{i j}-\lambda_{j}}{\lambda_{j}}, \quad \forall i \in S \cup\{0\} ; j \in S ; i \neq j
$$

and

$$
\begin{aligned}
U_{i j}^{R} & =\sup \left\{f_{(i, j) \mid P}\left(\lambda_{i \mid P}\right): \lambda_{i} \leq \lambda_{i \mid P} \leq 2 \lambda_{i}\right\} \\
& =f_{(i, j) \mid P}\left(2 \lambda_{i}\right)=\frac{\lambda_{i j}^{u}-\lambda_{j}}{\lambda_{j}}, \quad \forall i, j \in S ; i \neq j .
\end{aligned}
$$

(Note: The implications of notations $\lambda_{i j}$ and $\lambda_{i j}^{u}$ see Section 3.3.)

In order to construct the solvable model for CSIP, we introduce the "completely memoryless property" as described below.

That the RC effect has a completely memoryless property means that the investment region's $\mathrm{RC}$ effect on subsequent regions is limited to the immediate subsequent region. In other words, the completely memoryless property implies that most consumers will only notice the most recent region where this commodity or service has been in great demand or has been popular. According to the definitions of CFIs and sequential effectiveness, consider path $P, P \in \Omega_{N}$, where region $P_{i}$ 's sequential effectiveness $F_{P_{i} \mid P}$ depends on $\lambda_{P_{i-1} \mid P}$ only.

In addition, in this paper we only initially consider the case that RCCI for any pair $(i, j), \forall i \in S \cup\{0\} ; j \in S ; i \neq j$, approaches the value 1 (for the sake of model simplicity). That is, $\theta_{i j} \approx 1$ and thus the consumers in region $j$ are neither hot nor cool about following the behavior of the consumers in region $i$.

Lemma 1 Consider that regional characteristics effect has a completely memoryless property, then for any pair $\left(P_{i-1}, P_{i}\right)$ in path $P, P \in \Omega_{N}$,

$$
F_{P_{i} \mid P}=W_{P_{i-1}, P_{i}}^{R}+\frac{\lambda_{P_{i-1} \mid P}-\lambda_{P_{i-1}}}{\lambda_{P_{i-1}}} \cdot\left(U_{P_{i-1}, P_{i}}^{R}-W_{P_{i-1}, P_{i}}^{R}\right)
$$

Proof: Based on the definitions of the regional characteristics effect's completely memoryless property, it can be inferred that $\lambda_{P_{i} \mid P}=\lambda_{P_{i-1}, P_{i} \mid P}, i=1,2, \ldots, N$. Hence, it follows from Equation (6) that $F_{P_{i} \mid P}=\frac{\lambda_{P_{i-k}, P_{i} \mid P}-\lambda_{P_{i}}}{\lambda_{P_{i}}}$. Moreover, since the consumer in any region are neither fervent nor disinclined about following the behavior of the consumer in another investment region, we can know that $\theta_{P_{i-k}, P_{i}}=1, \forall k=1,2, \ldots, i-1 ; i=1,2, \ldots, N$.

According to Equations (7)-(8) and the above results, we can obtain that

$$
\begin{aligned}
& W_{P_{i-1}, P_{i}}^{R}+\frac{\lambda_{P_{i-1} \mid P}-\lambda_{P_{i-1}}}{\lambda_{P_{i-1}}} \cdot\left(U_{P_{i-1}, P_{i}}^{R}-W_{P_{i-1}, P_{i}}^{R}\right) \\
= & \frac{\lambda_{P_{i-1}, P_{i}}-\lambda_{P_{i}}}{\lambda_{P_{i}}}+\frac{\lambda_{P_{i-1}, P_{i} \mid P}-\lambda_{P_{i-1}, P_{i}}}{\lambda_{P_{i-1}, P_{i}}^{u}-\lambda_{P_{i-1}, P_{i}}} \times \frac{\lambda_{P_{i-1}, P_{i}}^{u}-\lambda_{P_{i-1}, P_{i}}}{\lambda_{P_{i}}} \quad \text { (By the results in Equation (2)) } \\
= & \frac{\lambda_{P_{i-1}, P_{i} \mid P}-\lambda_{P_{i}}}{\lambda_{P_{i}}}=F_{P_{i} \mid P .} .
\end{aligned}
$$

Hence, the result of this theorem is obtained.

In Lemma 1, the linear sequential effectiveness function of the regional characteristics effect can be illustrated by Figure 4 as shown below.

Remark 3: The implications of Lemma 2 include: (1) The consumption behavior in any investment region is very conservative and the demand rate for the product only increases 


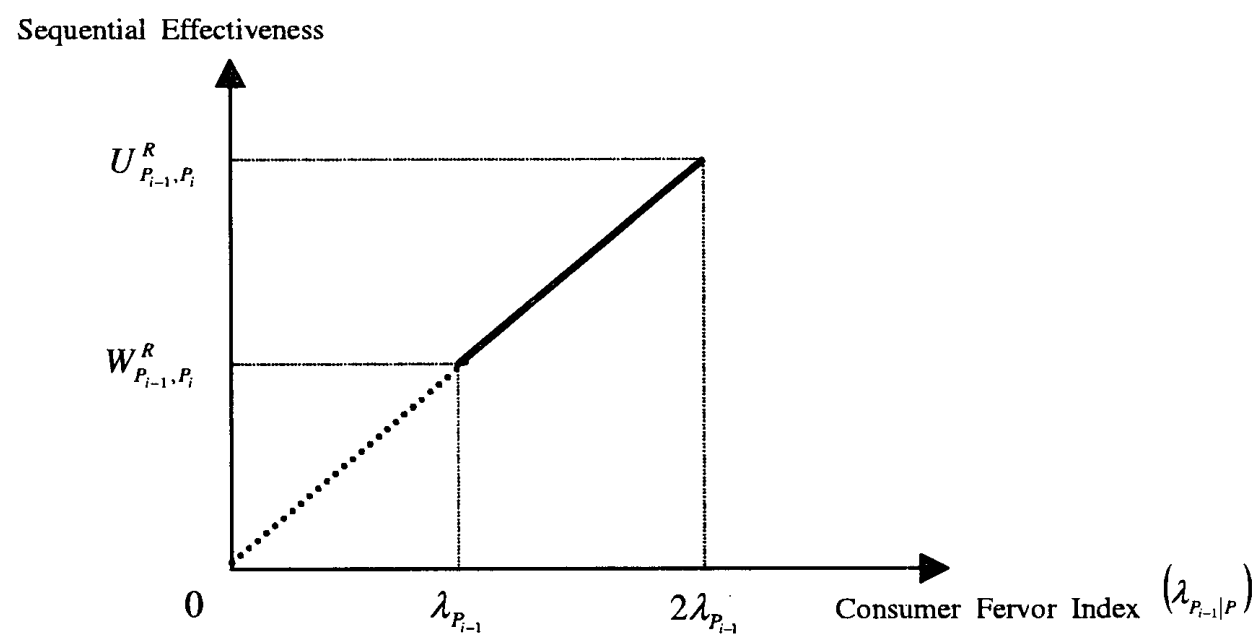

Figure 4: Linear function of regional characteristics effect

significantly when a great deal of many people buy the product (i.e., $\phi_{j} \approx 1, \forall j \in S$ ), and (2) The consumers in any investment region are neither fervent nor disinclined about following the behavior of the consumers in another investment region.

Lemma 2 Consider that regional characteristics effect has a completely memoryless property and that three-dimension-based sequential effectiveness can be ignored, then for any pair $\left(P_{i-1}, P_{i}\right)$ in path $P, P \in \Omega_{N}$

(1) $\quad F_{P_{i} \mid P} \approx W_{P_{i-1}, P_{i}}^{R}+\left(W_{P_{i-2}, P_{i-1}}^{R}\right) \cdot\left(U_{P_{i-1}, P_{i}}^{R}-W_{P_{i-1}, P_{i}}^{R}\right)$

(2) $\quad W_{\left(P_{i-2}, P_{i-1}\right) P_{i}}^{R}=W_{P_{i-2}, P_{i-1}}^{R} \cdot\left(U_{P_{i-1}, P_{i}}^{R}-W_{P_{i-1}, P_{i}}^{R}\right)$.

Proof: Since the RC effect has a completely memoryless property, we can find that $F_{P_{i} \mid P}=f_{\left(P_{i-1}, P_{i}\right) \mid P}\left(\lambda_{P_{i-1} \mid P}\right)$. Recall that $g_{\left(P_{i-k}, P_{i}\right) \mid P}^{j}$ represents the $(j+1)$-dimension-based sequential effectiveness. Since $g_{\left(P_{i-k}, P_{i}\right) \mid P}^{j}$ is strictly decreasing in $j$ (see Section 3.6), we can further omit the values of $\sum_{j \geq 3} g_{\left(P_{i-k}, P_{i}\right) \mid P}^{j}$ if we can omit the value of three-dimension-based sequential effectiveness. Therefore, we can obtain that $f_{\left(P_{i-1}, P_{i}\right) \mid P}\left(\lambda_{P_{i-1} \mid P}\right) \approx W_{P_{i-1}, P_{i}}^{R}+$ $W_{\left(P_{i-2}, P_{i-1}\right) P_{i}}^{R}$. Based on the above results and Equation (6), we can further obtain that $\lambda_{P_{i-1} \mid P} \approx \lambda_{P_{i-1}}+\left(W_{P_{i-2}, P_{i-1}}^{R}+W_{\left(P_{i-3}, P_{i-2}\right) P_{i-1}}^{R}\right) \lambda_{P_{i-1}}$. Hence, by Lemma 2 , we can find

$F_{P_{i} \mid P}=W_{P_{i-1}, P_{i}}^{R}+\left(\frac{\lambda_{P_{i-1}}+\left(W_{P_{i-2}, P_{i-1}}^{R}+W_{\left(P_{i-3}, P_{i-2}\right) P_{i-1}}^{R}\right) \lambda_{P_{i-1}}-\lambda_{P_{i-1}}}{\lambda_{P_{i-1}}}\right) \cdot\left(U_{P_{i-1}, P_{i}}^{R}-W_{P_{i-1}, P_{i}}^{R}\right)$

$=W_{P_{i-1}, P_{i}}^{R}+\left(W_{P_{i-2}, P_{i-1}}^{R}+W_{\left(P_{i-3}, P_{i-2}\right) P_{i-1}}^{R}\right) \cdot\left(U_{P_{i-1}, P_{i}}^{R}-W_{P_{i-1}, P_{i}}^{R}\right)$.

Since one element of $F_{P_{i} \mid P}$ is $W_{\left(P_{i-3}, P_{i-2}\right) P_{i-1}}^{R}\left(U_{P_{i-1}, P_{i}}^{R}-W_{P_{i-1}, P_{i}}^{R}\right), W_{\left(P_{i-3}, P_{i-2}\right) P_{i-1}}^{R}\left(U_{P_{i-1}, P_{i}}^{R}-\right.$ $\left.W_{P_{i-1}, P_{i}}^{R}\right)$ is an instance of three-dimension-based sequential effectiveness and can be ignored. According above results, we know that $F_{P_{i} \mid P} \approx W_{P_{i-1}, P_{i}}^{R}+W_{P_{i-2}, P_{i-1}}^{R} \cdot\left(U_{P_{i-1}, P_{i}}^{R}-W_{P_{i-1}, P_{i}}^{R}\right)$.

Moreover, since $F_{P_{i} \mid P} \approx W_{P_{i-1}, P_{i}}^{R}+W_{\left(P_{i-2}, P_{i}\right) P_{i}}^{R}$, we can obtain that $W_{\left(P_{i-2}, P_{i-1}\right) P_{i}}^{R}=$ $W_{P_{i-2}, P_{i-1}}^{R} \cdot\left(U_{P_{i-1}, P_{i}}^{R}-W_{P_{i-1}, P_{i}}^{R}\right)$. The result of this theorem is obtained.

Let $X_{i j}, \forall i, j \in S \cup\{0\}, i \neq j$, be the indicator variable when investment region $i$ achieves the target revenue and moves on to region $j$. In other words, if $X_{i j}=1$, after 
region $i$ achieves the target revenue, it moves on to region $j$; otherwise, $X_{i j}=0$. All the non-recurring directed paths of $S \cup\{0\}$ that starts at region 0 (the parent company) can be represented by constraint set $L$. If we define $\Lambda$ so that any vector $X \in \Lambda$ represents a non-recurring directed path that starts at region 0 ; i.e., $X_{i 0}=0, \forall i \in S$, then $\Lambda$ refers to $L$ 's feasible solution space.

Constraint set $L$ :

$\mathrm{C} 1: \sum_{i \in S \cup\{0\} ; i \neq j} X_{i j}=1, \forall j \in S$

$\mathrm{C} 2: \sum_{j \in S ; j \neq i} X_{i j} \leq 1, \forall i \in S$

C3: $\sum_{j \in S} X_{0 j}=1$

C4: $\sum_{j \in S ; j \neq i} \sum_{i \in S \cup\{0\}} X_{i j}=N$

C5: $X_{i_{1}, i_{2}}+X_{i_{2}, i_{3}}+\cdots+X_{i_{m-1}, i_{m}}+X_{i_{m}, i_{1}} \leq m-1$, $\forall i_{1}, i_{2}, \ldots, i_{m} \in S \cup\{0\} ; i_{1}, i_{2}, \ldots, i_{m}$ are all distinct, $m=2, \ldots, N$.

\section{Remark 4:}

(i) $\mathrm{C} 1$ means that just one investment region achieves its target revenue at the beginning of investment in region $j, \forall j \in S$.

(ii) $\mathrm{C} 2$ means that when an investment region $i$ (expect for $i$ is the final investment region) achieves its target revenue, the company will continue transfer the initial investment capital $\left(C_{0}\right)$ to another region.

(iii) C3 means that a feasible non-recurring directed path must start at region 0 (the parent company).

(iv) $\mathrm{C} 4$ refers to that capital $C_{0}$ is transferred $N$ times among $N$ planned investment regions.

(v) C5 means no recurrence is allowed in investment paths.

Theorem 3 Consider that the RC effect has a completely memoryless property and threedimension-based sequential effectiveness can be ignored, then CSIP can be formulated as:

$$
\begin{aligned}
\operatorname{Max}_{X \in A} Z= & \sum_{j \in S} \alpha_{j} W_{0 j}^{R} X_{0 j}+\sum_{k \in S, k \neq j} \sum_{j \in S} \alpha_{k} W_{0 j}^{R}\left(U_{j k}^{R}-W_{j k}^{R}\right) X_{0 j} X_{j k}+\sum_{i \in S, i \neq j} \sum_{j \in S, j \neq i} \alpha_{j} W_{i j}^{R} X_{i j} \\
& +\sum_{i \in S, i \neq j \neq k} \sum_{j \in S, j \neq k} \sum_{k \in S} \alpha_{k} W_{i j}^{R}\left(U_{j k}^{R}-W_{j k}^{R}\right) X_{i j} X_{j k} .
\end{aligned}
$$

Proof: From Lemma 3, we can obtain that

$$
\begin{aligned}
& \operatorname{Max}_{P \in \Omega_{N}} \sum_{P_{i} \in S} \alpha_{P_{i}} F_{P_{i} \mid P} \\
\approx & \operatorname{Max}_{P \in \Omega_{N}}\left\{\sum_{P_{i-1}, P_{i} \in S \cup\{0\}} \alpha_{P_{i}} W_{P_{i-1}, P_{i}}^{R}+\sum_{P_{i-2}, P_{i-1}, P_{i} \in S \cup\{0\}} \alpha_{P_{i}} W_{\left(P_{i-2}, P_{i-1}\right) P_{i}}^{R}\right\} \\
= & \operatorname{Max}_{P \in \Omega_{N}}\left\{\sum_{P_{i-1}, P_{i} \in S \cup\{0\}} \alpha_{P_{i}} W_{P_{i-1}, P_{i}}^{R}+\sum_{P_{i-2}, P_{i-1}, P_{i} \in S \cup\{0\}} \alpha_{P_{i}} W_{P_{i-2}, P_{i-1}}^{R} \cdot\left(U_{P_{i-1}, P_{i}}^{R}-W_{P_{i-1}, P_{i}}^{R}\right)\right\} \\
= & \operatorname{Max}_{P \in \Omega_{N}}\left\{\alpha_{P_{1}} W_{0, P_{1}}^{R}+\alpha_{P_{2}} W_{0, P_{1}}^{R} \cdot\left(U_{P_{1}, P_{2}}^{R}-W_{P_{1}, P_{2}}^{R}\right)+\sum_{P_{i-1}, P_{i} \in S} \alpha_{P_{i}} W_{P_{i-1}, P_{i}}^{R}\right. \\
& \left.+\sum_{P_{i-2}, P_{i-1}, P_{i} \in S} \alpha_{P_{i}} W_{P_{i-2}, P_{i-1}}^{R} \cdot\left(U_{P_{i-1}, P_{i}}^{R}-W_{P_{i-1}, P_{i}}^{R}\right)\right\}
\end{aligned}
$$




$$
\begin{aligned}
=\operatorname{Max}_{P \in \Omega_{N}}\left\{\alpha_{P_{1}} W_{0, P_{1}}^{R} X_{0, P_{1}}+\alpha_{P_{2}} W_{0, P_{1}}^{R} \cdot\left(U_{P_{1}, P_{2}}^{R}-W_{P_{1}, P_{2}}^{R}\right) X_{0, P_{1}} X_{P_{1}, P_{2}}+\sum_{P_{i-1}, P_{i} \in S} \alpha_{P_{i}} W_{P_{i-1}, P_{i}}^{R} X_{P_{i-1}, P_{i}}\right. \\
\left.+\sum_{P_{i-2}, P_{i-1}, P_{i} \in S} \alpha_{P_{i}} W_{P_{i-2}, P_{i-1}}^{R} \cdot\left(U_{P_{i-1}, P_{i}}^{R}-W_{P_{i-1}, P_{i}}^{R}\right) X_{P_{i-2}, P_{i-1}} X_{P_{i-1}, P_{i}}\right\} \\
=\operatorname{Max}_{X \in \Lambda} \sum_{j \in S} \alpha_{j} W_{0 j}^{R} X_{0 j}+\sum_{k \in S, k \neq j} \sum_{j \in S}\left(\alpha_{k} W_{0 j}^{R}\left(U_{j k}^{R}-W_{j k}^{R}\right)\right) X_{0 j} X_{j k}+\sum_{i \in S, i \neq j} \sum_{j \in S} \alpha_{j} W_{i j}^{R} X_{i j} \\
\quad+\sum_{i \in S, i \neq j \neq k} \sum_{j \in S, j \neq k} \sum_{k \in S}\left(\alpha_{k} W_{i j}^{R}\left(U_{j k}^{R}-W_{j k}^{R}\right)\right) X_{i j} X_{j k} .
\end{aligned}
$$

Theorem 3 is derived as show above.

\section{Remark 5:}

(i) If a company plans to market a new product, then the parent company's location can be one of the planned investment regions. In this case, the values of $W_{0 j}^{R}$ and $U_{0 j}^{R}$ are set to be zero for all $j$ belonging to $S$.

(ii) Consider the special case that CSIP can formulated as $\operatorname{Max}_{P \in \Omega_{N}}\left\{\alpha_{P_{1}} W_{0, P_{1}}^{R}+\sum_{i=2}^{N} \alpha_{P_{i}} W_{P_{i-1}, P_{i}}^{R}\right\}$. We can easy to demonstrate that $\operatorname{Max}_{P \in \Omega_{N}} \sum_{i=1}^{N} \alpha_{P_{i}} W_{P_{i^{\prime}}, P_{i}}^{R} \cong \operatorname{Min}_{P \in \Omega_{N}} \sum_{i=1}^{N}\left(1-\alpha_{P_{i}} W_{P_{i-1}, P_{i}}^{R}\right)$. Consider an asymmetric traveling salesman problem (ATSP) including $L$ cities which labels by number $1,2, \ldots, L$. Also, define $\left[d_{i j}\right]$ be the distance matrix $\left(d_{i j} \neq 0\right)$. We call this ATSP $Y$. Moreover, consider another ATSP case including $2 L$ cities which labels by number $1,1^{\prime}, 2,2^{\prime}, \ldots, L, L^{\prime}$. Also, define $\left[\bar{d}_{l m}\right]$ be the distance matrix, where $\bar{d}_{i^{\prime} i}=\bar{d}_{i i^{\prime}}=0$, and $\bar{d}_{i^{\prime} j}=\bar{d}_{i j^{\prime}}=\bar{d}_{i^{\prime} j^{\prime}}=d_{i j}$. We call this ATSP $Z$. Obviously, ATSP $Y$ is equivalent to $\operatorname{ATSP} Z$. For ATSP $Z$, if we choose two cities $i$ and $i^{\prime}$, and view city $i^{\prime}$ as the parent company and $i$ as final investment region, then we can solve ATSP $Z$ by finding a nonrecurring directed path (since $\bar{d}_{i i^{\prime}}=0$ ). That is, ATSP $Z$ is transformed into the above special case of CSIP. In other words, the ATSP reduces to the CSIP. Since any instance of ATSP without the special structured distance matrix is NP-hard, thus any instance of CSIP is also NP-hard.

\section{Parameters Estimation}

For further study of our model's parameters, the relevant symbols are defined as follows:

$\widetilde{M}_{j}(t)=$ A buyer's average consumption level when he/she arrives to buy the commodity at time $t, \forall j \in S \cup\{0\}$.

$\widetilde{\beta}_{j}(t)=$ The average arrival rate at time $t$ for buyers, $\forall j \in S \cup\{0\}$.

$\widetilde{N}_{j \mid P}(t)=$ The expected consumer population up to time $t$ when region $j$, under the influence of the regional characteristics of prior invested regions, achieves the target revenue $\left(R_{j}\right), \forall j \in S \cup\{0\} ; P \in \Omega_{N}$.

$\beta_{j}(y)=$ The average arrival rate at a time for buyers when total consumer population up to that time is $y, \forall j \in S \cup\{0\}$.

$M_{j}(x)=$ The expected consumption level at a time when the arrival rate at that time is $x, \forall j \in S \cup\{0\}$. 
$N_{j \mid P}=$ The expected consumer population when region $j$, under the influence of the regional characteristics of prior invested regions, achieves the target revenue $\left(R_{j}\right), \forall j \in S \cup\{0\} ; P \in \Omega_{N}$.

$N_{j}=$ The expected consumer population when region $j$, uninfluenced by the previous investment areas, achieves the target revenue $\left(R_{j}\right), \forall j \in S \cup\{0\}$.

$N_{j}^{u}=$ The expected consumer population that enables region $j$ to achieve the target revenue $\left(R_{j}\right)$ within time $\varepsilon_{j}$, where $\varepsilon_{j}$ is a miniscule number relative to $E\left[T_{j}\right], \forall j \in S$

$N_{i j}=$ The expected consumer population when region $j$, under the influence of the regional characteristics of region $i$, achieves the target revenue $\left(R_{j}\right)$ and the consumer population in region $i$ reaches $N_{i}, \forall i \in S \cup\{0\} ; j \in S ; i \neq j$.

$N_{i j}^{u}=$ The expected consumer population when the consumer population in region $i$ reaches $N_{i}^{u}$ and region $j$, under the influence of the regional characteristics of region $i$, achieves the target revenue $\left(R_{j}\right), \forall i \in S \cup\{0\} ; j \in S ; i \neq j$.

From the definition of CFI, we can intuitively obtain that both and $\widetilde{M}_{j}\left(E\left[T_{j \mid P}\right]\right)$ and $\widetilde{\beta}_{j}\left(E\left[T_{j \mid P}\right]\right)$ are the major parameters to define $\lambda_{j \mid P}$. In this paper we initially suppose that the consumption level at any time only depends on the arrival rate at that time, i.e., $\widetilde{M}_{j}(t)$ only depends on arrival rate $\widetilde{\beta}_{j}(t)$. We also suppose that the arrival rate $\widetilde{\beta}_{j}(t)$ only depends on consumer population $\widetilde{N}_{j \mid P}(t)$. Therefore, we obtain that

$$
\tilde{\beta}_{j}\left(E\left[T_{j \mid P}\right]\right)=\beta_{j}\left(N_{j \mid P}\right)
$$

and

$$
\widetilde{M}_{j}\left(E\left[T_{j \mid P}\right]\right)=M_{j}\left(\widetilde{\beta}_{j}\left(E\left[T_{j \mid P}\right]\right)\right)=M_{j}\left(\beta_{j}\left(N_{j \mid P}\right)\right) .
$$

According to Equations (12) and (13), we can assume that all information related to $\widetilde{M}_{j}\left(E\left[T_{j \mid P}\right]\right)$ and $\widetilde{\beta}_{j}\left(E\left[T_{j \mid P}\right]\right)$ for defining $\lambda_{j \mid P}$ will be involved in $N_{j \mid P}$. As a result, in this paper we use $N_{j \mid P}$ to define $\lambda_{j \mid P}$. We can define $\lambda_{j \mid P}$ as follows:

$$
\frac{\lambda_{j \mid P}-\lambda_{j}}{2 \lambda_{j}-\lambda_{j}}=\frac{N_{j \mid P}-N_{j}}{N_{j}^{u}-N_{j}}, \forall j \in S \cup\{0\} ; P \in \Omega_{N}
$$

Therefore, Equations (7) and (8) can be rewritten as follows:

$$
W_{i j}^{R}=\frac{N_{i j}-N_{j}}{N_{j}^{u}-N_{j}}, \forall i \in S \cup\{0\} ; j \in S ; i \neq j
$$

and

$$
U_{i j}^{R}=\frac{N_{i j}^{u}-N_{j}}{N_{j}^{u}-N_{j}}, \forall i, j \in S ; i \neq j .
$$

We further define four types of consumers as described below.

Type 1: We say that a consumer is type 1 if he/she would became a buyer due to the inherent attractiveness of the commodity.

Type 2: We say that a consumer is type 2 if he/she would not have bought the product but became a buyer when they were influenced by an ingenious promotion scheme.

Type 3: We say that a consumer is type 3 if he/she would not have bought the product but became a buyer due to the influence of the fervent buying of prior invested regions.

Type 4: Otherwise.

Supposing the consumers of the commodity for each investment region can be classified into one of the four types above, we then further define extra notations as follows: 
$\widetilde{N}_{i j \mid P}(t)=$ The expected consumer population up to time $t$ when we take into account the RC effect that region $i$ has on region $j, \forall i \in S \cup\{0\} ; j \in S ; P \in \Omega_{N}$.

$\tilde{a}_{i j \mid P}(t)=$ The expected increase in consumer population up to time $t$ that can be attributed to the emergence of consumers who would not have bought the product but became buyers when they were influenced by the fervent buying of region $i$ (i.e., type 3 consumers), $\forall i \in S \cup\{0\} ; j \in S ; P \in \Omega_{N}$.

$\tilde{b}_{j}(t)=$ The expected consumer population of type 1 produced up to time $t, \forall j \in$ $S \cup\{0\}$.

$\widetilde{c}_{j}(t)=$ The expected consumer population of type 2 produced up to time $t, \forall j \in$ $S \cup\{0\}$.

$\tilde{d}_{j}(t)=$ The expected consumer population of type 4 produced up to time $t, \forall j \in$ $S \cup\{0\}$.

According to the above notations, we further suppose that

$$
\begin{aligned}
\widetilde{N}_{i j \mid P}(t) & \approx \tilde{a}_{i j \mid P}(t)+\tilde{b}_{j}(t)+\tilde{c}_{j}(t)+\tilde{d}_{j}(t) \\
& \approx a_{i j \mid P}+b_{j}+c_{j}+d_{j} \quad \text { if } t \geq \bar{t}_{j} .
\end{aligned}
$$

Recalling for any investment region $j \forall j \in S \cup\{0\}$, that region $j$ achieving its target revenue $\left(R_{j}\right)$ means that the corporation in region $j$ has developed such an operational capacity that it can maintain stable operation (see Section 1). Hence, we can intuitively consider the case that $E\left[T_{j \mid P}\right]>\bar{t}_{j}$ (because target revenue $R_{j}$ is usually several times of capital $C_{0}$ and thus $E\left[T_{j \mid P}\right]$ is usually very long), i.e., the consumer population buying behavior (for all types of consumers) produced before the target revenue is achieved. Accordingly, we can find:

$$
\begin{aligned}
N_{j} & \approx b_{j}+c_{j}+d_{j} \\
N_{i j} & \approx a_{i j}+b_{j}+c_{j}+d_{j}
\end{aligned}
$$

and

$$
N_{i j}^{u} \approx a_{i j}^{u}+b_{j}+c_{j}+d_{j}
$$

Where $a_{i j}$ and $a_{i j}^{u}$ denote the expected consumer populations of type 3 when region $j$ achieves the target revenue $\left(R_{j}\right)$, when the consumer populations in region $i$ have already reached $N_{i}$ and $N_{i}^{u}$, respectively.

Moreover, let $\beta_{j}^{u}$ be average maximum arrival rate for buyers in region $j$, and let $m_{j}^{u}$ be the average consumption level when $\beta_{j}^{u}$ is achieved. Consider the case that there exists a consumer population $z_{j}, \forall j \in S \cup\{0\}$ so that

$$
\beta_{j}(y)=\beta_{j}^{u}, \quad \text { if } y \geq z_{j} .
$$

Hence,

$$
M_{j}(x)=m_{j}^{u}, \quad \text { if } x \geq z_{j} .
$$

If we consider the case that $\frac{R_{j}}{m_{j}^{u} \cdot \beta_{j}^{u}} \ll E\left[T_{j}\right], \forall j \in S \cup\{0\}$, then by Equations (18) and (19), we can set

$$
N_{j}^{u}=z_{j}, \forall j \in S
$$

We can obtain the values of $a_{i j}^{u}, z_{j}, b_{j}, c_{j}$, and $d_{j}$ via a careful survey and evaluation of market, $\forall i \in S ; j \in S \cup\{0\} ; i \neq j$. 
In the following we will introduce a simple method to obtain the values of $a_{i j}, \forall i \in$ $S \cup\{0\} ; j \in S ; i \neq j$. Let $N_{i j \mid P}$ be the expected consumer population when region $j$ achieves the target revenue $\left(R_{j}\right)$ when consumer population in region $i$ is $N_{i \mid P}, \forall i \in S \cup\{0\} ; P \in \Omega_{N}$. According to Equations (17) through (20) we can find

$$
\frac{N_{i j \mid P}-N_{i j}}{N_{i j}^{u}-N_{i j}} \approx \frac{\left(a_{i j \mid P}+b_{j}+c_{j}\right)-\left(a_{i j}+b_{j}+c_{j}\right)}{\left(a_{i j}^{u}+b_{j}+c_{j}\right)-\left(a_{i j}+b_{j}+c_{j}\right)}=\frac{a_{i j \mid P}-a_{i j}}{a_{i j}^{u}-a_{i j}} .
$$

In addition, consider an ordered pair $(i, j)$ in path $P, P \in \Omega_{N}$, since $\frac{N_{i j \mid P}-N_{i j}}{N_{i j}^{u}-N_{i j}} \approx\left(\frac{N_{i \mid P}-N_{i}}{N_{i}^{u}-N_{i}}\right)^{\theta_{i j}}$ (by Equations (2) and (14)), we can find:

$$
\frac{a_{i j \mid P}-a_{i j}}{a_{i j}^{u}-a_{i j}} \approx\left(\frac{N_{i \mid P}-N_{i}}{N_{i}^{u}-N_{i}}\right)^{\theta_{i j}}, \forall i \in S ; j \in S \cup\{0\} ; i \neq j .
$$

Remark 6: When $\theta_{i j}<1, a_{i j \mid P}$ and $N_{i \mid P}$ in the range $\left[N_{i}, N_{i}^{u}\right]$ approximate a nonlinear concave function. When $\theta_{i j}>1, a_{i j \mid P}$ and $N_{i \mid P}$ in the range $\left[N_{i}, N_{i}^{u}\right]$ approximate a nonlinear convex function. When $\theta_{i j}=1, a_{i j \mid P}$ and $N_{i \mid P}$ in the range $\left[N_{i}, N_{i}^{u}\right]$ approximate a linear function. The implications of $\theta_{i j}, \forall i \in S \cup\{0\} ; j \in S ; i \neq j$ can be found in Section 3.3 in this paper. The relationship between $a_{i j \mid P}$ and $N_{i \mid P}$ is illustrated in Figure 5 as follows:

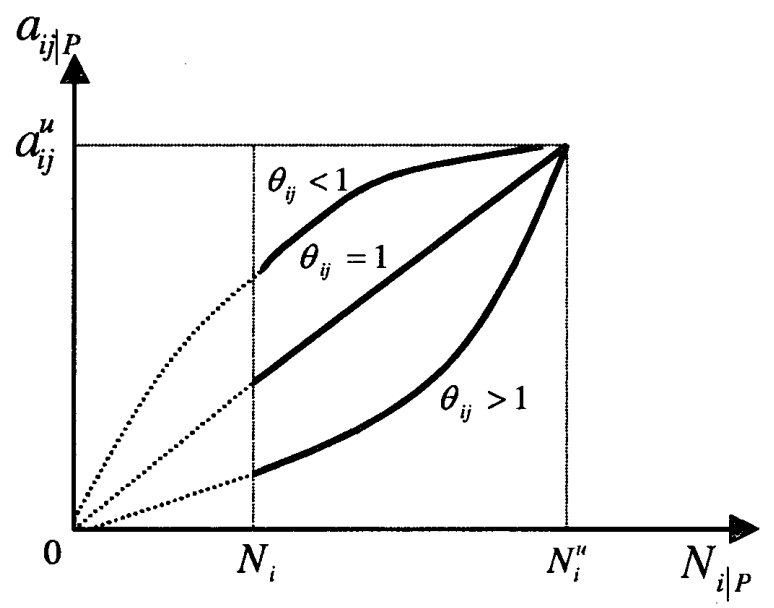

Figure 5: The relationship between $a_{i j \mid P}$ and $N_{i \mid P}$

Since $\theta_{i j}$ depends on $N_{i}$, we use the symbol $\theta_{i j}\left(N_{i}\right)$ instead of $\theta_{i j}, \forall i \in S ; j \in S \cup\{0\}$; $i \neq j$, thus, Equation (25) can be rewritten as follows:

$$
\frac{a_{i j \mid P}-a_{i j}}{a_{i j}^{u}-a_{i j}} \approx\left(\frac{N_{i \mid P}-N_{i}}{N_{i}^{u}-N_{i}}\right)^{\theta_{i j}\left(N_{i}\right)}, \forall i \in S \cup\{0\} ; j \in S ; i \neq j .
$$

If we believe that the consumers in investment region $j$ are neither fervent nor disinclined about following the behavior of consumers in region $i$ (see Section 3.3), then we can obtain that $\theta_{i j}\left(N_{i}\right)=1$ for all $N_{i}$ on the range $\left[0, N_{i}^{u}\right]$. Therefore, we can easy obtain $a_{i j}$ by using the following formula:

$$
\frac{a_{i j}}{a_{i j}^{u}} \approx \frac{N_{i}}{N_{i}^{u}}, \forall i \in S \cup\{0\} ; j \in S ; i \neq j
$$




\section{Genetic Algorithm-Based Procedure}

The genetic algorithm was proposed by John Holland in 1975 [6]. It is a general search technique which has a parallel property and the ability to avoid falling into regional optimization. This algorithm searches for the optimal solution using natural selection and natural inheritance, control over the fittest for survival, and stochastic exchange of information. Hence, every generation will produce a new solution set that suits the fitness of the problems of its era better [4]. This is a popular heuristic algorithm of stochastic search and has been used in solving many NP-Hard optimization problems [11]. Since there are well-developed user-friendly softwares available which can effectively solve genetic algorithms (Evolver) and which are also easy to use and achieve good performance $[1,7,8,9,10,13,14]$. This research uses genetic algorithms as the main problem-solving tool. The algorithm procedure developed in this research by integrating genetic algorithms is described in Figure 6 as follows:

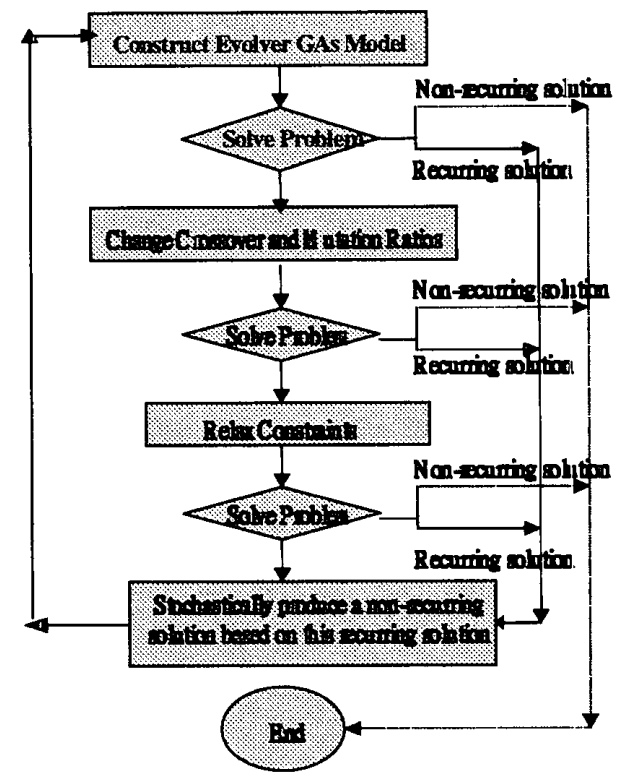

Figure 6: Integrating genetic algorithm flowchart

Step 1. Construct the following objective function using Evolver software:

$$
\begin{aligned}
Z= & \sum_{j \in S} \alpha_{j} W_{0 j}^{R} X_{0 j}+\sum_{k \in S, k \neq j} \sum_{j \in S}\left(\alpha_{k} W_{0 j}^{R}\left(U_{j k}^{R}-W_{j k}^{R}\right)\right) X_{0 j} X_{j k}+\sum_{i \in S, i \neq j} \sum_{j \in S} \alpha_{j} W_{i j}^{R} X_{i j} \\
& +\sum_{i \in S, i \neq j \neq k} \sum_{j \in S, j \neq k} \sum_{k \in S}\left(\alpha_{k} W_{i j}^{R}\left(U_{j k}^{R}-W_{j k}^{R}\right)\right) X_{i j} X_{j k} .
\end{aligned}
$$

Step 2. Define the following constraints in Evolver software:

$$
\begin{aligned}
& X_{i j}+X_{i j} \leq 1, \forall i, j \in S \cup\{0\} ; i \neq j \\
& \sum_{i \in S \cup\{0\} ; i \neq j} X_{i j}=1, \forall j \in S \\
& \sum_{j \in S ; j \neq i} X_{i j} \leq 1, \forall i \in S \\
& \sum_{j \in S} X_{0 j}=1
\end{aligned}
$$




$$
\sum_{j \in S ; j \neq i} \sum_{i \in S \cup\{0\}} X_{i j}=N
$$

Step 3. Execute Evolver to solve the problem.

Step 3.1. If no solution is obtained after running for a significant period of time, increase the matching or mutation rate in order to expand the search space for solution.

Step 3.2. If no solution is obtained within the preset period of time after adjusting the parameters (matching rate or mutation rate), then relax constraints. For example, change constraint $\sum_{j \in S ; j \neq i} \sum_{i \in S \cup\{0\}} X_{i j}=N$ to $\sum_{j \in S ; i \neq j} \sum_{i \in S \cup\{0\}} X_{i j} \leq 2 N$.

Step 4. Act on the final solution obtained.

Step 4.1. If the final solution obtained is non-recurring, then end.

Step 4.2. If the final solution obtained is recurring, then execute Step 5.

Step 5. Based on the recurring solution obtained in Step 4.2, produce a feasible solution by the following procedure and avoid producing the same recurring solution by controlling the "adjustable variables" in Evolver.

Case 1: If there exists more than one recurrence then generate a new initial solution as described below. If we let the original initial solution be $X_{0, i_{1}}=X_{i_{1}, i_{2}}=\cdots=$ $X_{i_{N-1}, i_{N}}=1$, then generate a new initial solution by setting $X_{0, i_{N}}=X_{i_{N}, i_{2}}=$ $\cdots=X_{i_{N-1}, i_{1}}=1, i_{1}, i_{2}, \ldots, i_{N}$ are all distinct.

Case 2: If there exists only one recurrence then generate a new initial solution as desribed below. Let $G$ be the link set consisting of a recurring sub-path in the current solution and $\bar{G}$ be the link set consisting of a non-recurring sub-path in the current solution. If link $(i, j)$ and $(k, i)$ belong to $G$ and link $(m, l)$ belongs to $\bar{G}$, then set $X_{k i}=0$ and $X_{k m}=1$.

For instance, in the problem involving six investment regions, assume the final solution obtained from Step 1 to Step 2 is: $1 \rightarrow 2 \rightarrow 3 \rightarrow 4 \rightarrow 1$ and $5 \rightarrow 6$. This solution can be written by $0-1$ variable as: $X_{12}=1, X_{23}=1, X_{34}=1, X_{41}=1, X_{56}=1$, other variables are zero. Based on above procedure, we can set $X_{41}=0$ and $X_{45}=1$ and then produce a new initial feasible solution are as $X_{12}=1, X_{23}=1, X_{34}=1, X_{41}=1, X_{56}=1$. We can use this solution as the initial feasible solution, then consider $X_{41}$ to be a variable that is not adjustable (in the Evolver) so as to avoid repetition of the same recurring solution.

Step 6. Repeat Step 3 to Step 5 until an approximately optimal feasible solution is obtained.

Step 3.1 shows that lower matching or mutation rates can be used in the early stage of problem-solving and then can be gradually increased when no reasonable solution is obtained. Mutation ratio needs micro adjustment and must be controlled within a certain range; otherwise it may adversely affect results.

Step 3.2 shows that in solving the model in this research, the "Pause" function of Evolver software can be used to monitor any change in problem-solving in a timely manner. The reasonable solution is first obtained under relaxed conditions, and then recalculated as adjustable variables decrease and constraints increase. The process continues until a set of optimal reasonable solutions are finally found satisfying the constraints in Step 2.

Step 4.2 explains that the optimal solution satisfying constraints in Step 2 is not necessarily a non-recurring directed path. Therefore, the method described in Step 5 is used in this research to promptly adjust this reasonable solution in a micro manner in order to obtain an optimal non-recurring directed path. This solution provides an initial reasonable value for calculating the optimal solution by executing Evolver software. 


\section{Illustrative Example}

Assume a transnational corporation known worldwide realizes that the motorcycle industry has great potential due to the trend of environmental protection. In order to take the first move to obtain the leading position in international market, the company plans to adopt different market entry modes such as wholly-owned venture and acquiring controlling interest in nine investment regions around the world, with the "centralized sequential investment strategy" defined in this research as its operational mode. These areas are coded by number 1 to 9 , where

$$
\begin{array}{r}
1 \equiv \text { Tokyo, } 2 \equiv \text { Rome, } 3 \equiv \text { Shanghai, } 4 \equiv \text { Chicago, } 5 \equiv \text { Paris } \\
6 \equiv \text { Vienna, } 7 \equiv \text { Milan, } 8 \equiv \text { Vancouver, } 9 \equiv \text { Osaka }
\end{array}
$$

This is not an empirical example, so a set of stochastically simulated parameters are used, without consideration of the parent company's influence (i.e., set $W_{0 j}^{R}=U_{0 j}^{R}=0$ ), to illustrate the procedure of applying genetic algorithms to obtain the final solution as shown below.

Table 1: Estimated values of $W_{i j}^{R}$

\begin{tabular}{cccccccc}
\hline$(1,2)$ & $(1,3)$ & $(1,4)$ & $(1,5)$ & $(1,6)$ & $(1,7)$ & $(1,8)$ & $(1,9)$ \\
0.091592 & 0.164548 & 0.288656 & 0.249722 & 0.083195 & 0.219354 & 0.037409 & 0.254038 \\
$(2,1)$ & $(2,3)$ & $(2,4)$ & $(2,5)$ & $(2,6)$ & $(2,7)$ & $(2,8)$ & $(2,9)$ \\
0.169832 & 0.285594 & 0.180317 & 0.058387 & 0.185664 & 0.065648 & 0.020352 & 0.142751 \\
$(3,1)$ & $(3,2)$ & $(3,4)$ & $(3,5)$ & $(3,6)$ & $(3,7)$ & $(3,8)$ & $(3,9)$ \\
0.116184 & 0.206574 & 0.225958 & 0.101092 & 0.191607 & 0.236845 & 0.222294 & 0.11532 \\
$(4,1)$ & $(4,2)$ & $(4,3)$ & $(4,5)$ & $(4,6)$ & $(4,7)$ & $(4,8)$ & $(4,9)$ \\
0.125792 & 0.066441 & 0.083441 & 0.240664 & 0.223762 & 0.125494 & 0.141927 & 0.126546 \\
$(5,1)$ & $(5,2)$ & $(5,3)$ & $(5,4)$ & $(5,6)$ & $(5,7)$ & $(5,8)$ & $(5,9)$ \\
0.270119 & 0.003469 & 0.231704 & 0.132335 & 0.128313 & 0.221839 & 0.141389 & 0.240726 \\
$(6,1)$ & $(6,2)$ & $(6,3)$ & $(6,4)$ & $(6,5)$ & $(6,7)$ & $(6,8)$ & $(6,9)$ \\
0.238205 & 0.071674 & 0.17796 & 0.003658 & 0.288782 & 0.28972 & 0.200307 & 0.181218 \\
$(7,1)$ & $(7,2)$ & $(7,3)$ & $(7,4)$ & $(7,5)$ & $(7,6)$ & $(7,8)$ & $(7,9)$ \\
0.205181 & 0.043608 & 0.239617 & 0.143476 & 0.045987 & 0.198969 & 0.241502 & 0.05321 \\
$(8,1)$ & $(8,2)$ & $(8,3)$ & $(8,4)$ & $(8,5)$ & $(8,6)$ & $(8,7)$ & $(8,9)$ \\
0.041181 & 0.098797 & 0.15084 & 0.061509 & 0.245278 & 0.242806 & 0.260475 & 0.085657 \\
$(9,1)$ & $(9,2)$ & $(9,3)$ & $(9,4)$ & $(9,5)$ & $(9,6)$ & $(9,7)$ & $(9,8)$ \\
0.10569 & 0.041852 & 0.09502 & 0.250967 & 0.007929 & 0.235224 & 0.201075 & 0.218389 \\
\hline
\end{tabular}

Based on the parameters in Table 1, Table 2, and Table 3, apply genetic algorithms and the problem-solving procedure proposed in this research. The program is executed a total of 3180 times, among which 1578 feasible solutions are generated in 25 seconds. Finally, the approximately optimal solution obtained is as follows:

$$
X_{23}=X_{34}=X_{49}=X_{96}=X_{67}=X_{78}=X_{85}=X_{51}=1 .
$$

All the other variables are zero; hence, the objective function $Z=0.3374$, meaning if $E\left[\sum_{P_{i} \in S} T_{P_{i}}\right]$ is the original estimated time for all the investment regions to achieve target revenues, then the transnational investment that follows from this sequence will shorten the 
Table 2: Estimated values of $U_{i j}^{R}$

\begin{tabular}{cccccccc}
\hline$(1,2)$ & $(1,3)$ & $(1,4)$ & $(1,5)$ & $(1,6)$ & $(1,7)$ & $(1,8)$ & $(1,9)$ \\
0.462129 & 0.598425 & 0.578272 & 0.451079 & 0.493609 & 0.579577 & 0.471788 & 0.4714 \\
$(2,1)$ & $(2,3)$ & $(2,4)$ & $(2,5)$ & $(2,6)$ & $(2,7)$ & $(2,8)$ & $(2,9)$ \\
0.458665 & 0.586996 & 0.52873 & 0.501606 & 0.517722 & 0.590188 & 0.47382 & 0.427681 \\
$(3,1)$ & $(3,2)$ & $(3,4)$ & $(3,5)$ & $(3,6)$ & $(3,7)$ & $(3,8)$ & $(3,9)$ \\
0.500312 & 0.407765 & 0.504378 & 0.535963 & 0.532834 & 0.401478 & 0.561618 & 0.48929 \\
$(4,1)$ & $(4,2)$ & $(4,3)$ & $(4,5)$ & $(4,6)$ & $(4,7)$ & $(4,8)$ & $(4,9)$ \\
0.556993 & 0.465771 & 0.575273 & 0.598491 & 0.426582 & 0.504932 & 0.575527 & 0.49188 \\
$(5,1)$ & $(5,2)$ & $(5,3)$ & $(5,4)$ & $(5,6)$ & $(5,7)$ & $(5,8)$ & $(5,9)$ \\
0.599855 & 0.42202 & 0.498683 & 0.411704 & 0.58647 & 0.566878 & 0.555466 & 0.477124 \\
$(6,1)$ & $(6,2)$ & $(6,3)$ & $(6,4)$ & $(6,5)$ & $(6,7)$ & $(6,8)$ & $(6,9)$ \\
0.474218 & 0.538591 & 0.544646 & 0.408808 & 0.544951 & 0.407395 & 0.469426 & 0.473133 \\
$(7,1)$ & $(7,2)$ & $(7,3)$ & $(7,4)$ & $(7,5)$ & $(7,6)$ & $(7,8)$ & $(7,9)$ \\
0.482431 & 0.594149 & 0.577046 & 0.414869 & 0.47321 & 0.437799 & 0.470301 & 0.470255 \\
$(8,1)$ & $(8,2)$ & $(8,3)$ & $(8,4)$ & $(8,5)$ & $(8,6)$ & $(8,7)$ & $(8,9)$ \\
0.41783 & 0.555154 & 0.406307 & 0.580677 & 0.472282 & 0.524958 & 0.453541 & 0.468483 \\
$(9,1)$ & $(9,2)$ & $(9,3)$ & $(9,4)$ & $(9,5)$ & $(9,6)$ & $(9,7)$ & $(9,8)$ \\
0.415731 & 0.540708 & 0.538252 & 0.416077 & 0.537215 & 0.426103 & 0.598868 & 0.522658 \\
\hline
\end{tabular}

Table 3: Estimated values of $\alpha_{i}$

\begin{tabular}{ccccccccc}
\hline$\alpha_{1}$ & $\alpha_{2}$ & $\alpha_{3}$ & $\alpha_{4}$ & $\alpha_{5}$ & $\alpha_{6}$ & $\alpha_{7}$ & $\alpha_{8}$ & $\alpha_{9}$ \\
0.14 & 0.07 & 0.11 & 0.14 & 0.09 & 0.18 & 0.08 & 0.11 & 0.08 \\
\hline
\end{tabular}

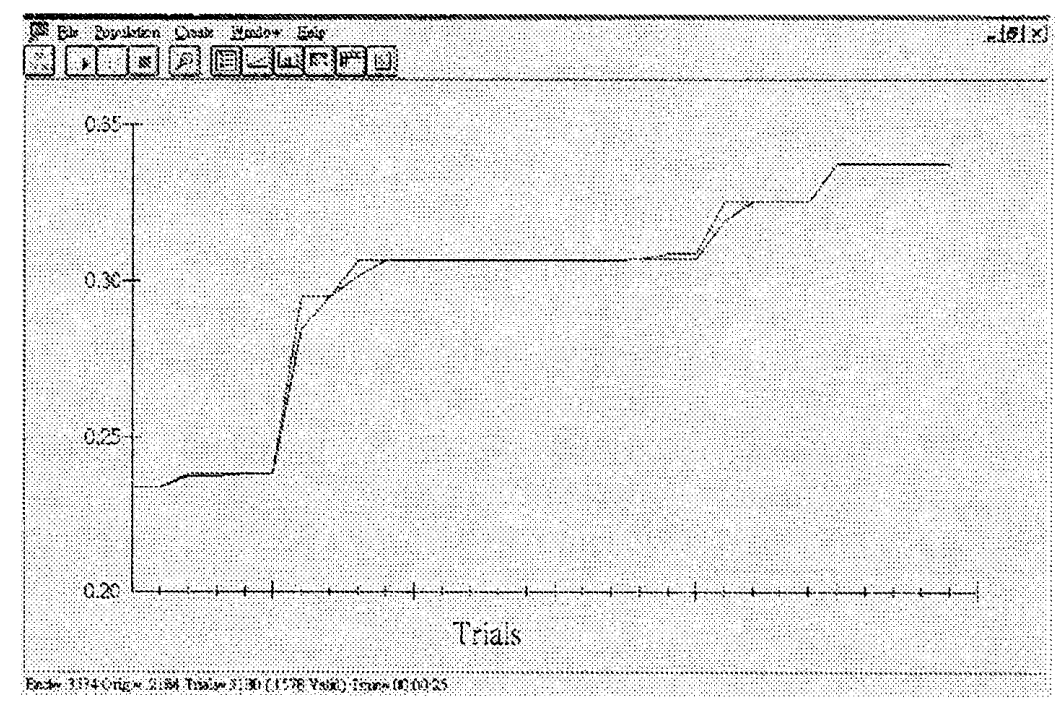

Figure 7: The converging chart of problem-solving procedure

original estimated time by $33.74 \%$. The converging chart of the problem-solving procedure in this example is shown in Figure 7. The investment sequence can be graphed by real regions as show in Figure 8. 


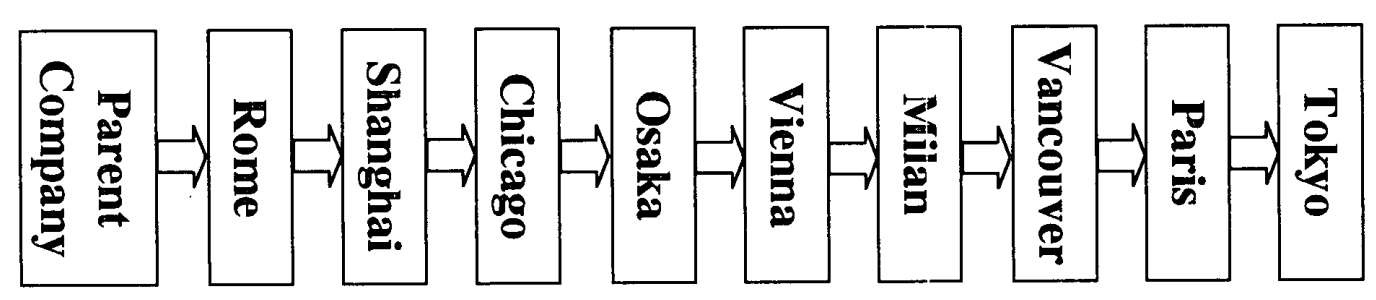

Figure 8: Investment sequence chart Described by real regions

\section{Conclusions}

Based on the introduction of the centralized sequential investment strategy, this paper proposed an optimization model for centralized sequential investment problems (CSIP), and discovered the optimal sequential investment path leading to achievement of target revenues for all investment regions within the shortest period of time. Furthermore, we assumed regional characteristics were a significant factor that affected the timing of reaching the target revenue in an investment region, and introduced the concepts of path effectiveness and sequential effectiveness. The original problem of "minimizing the expected time to achieve the target revenues for all investment regions" was then converted into the problem of "maximizing the sum of all regions' weighted sequential effectiveness". As a result, further properties of CSIP were demonstrated and thus formulated into a solvable form. We also proposed a simple method to estimate the model parameters. Finally, a GA-based solution procedure was proposed and a numerical example was illustrated. In this paper we supposed that the $\mathrm{RC}$ effect had a completely memoryless property and only considered the linear $\mathrm{RC}$ effect case; hence, further research should be focused on closing this gap. In addition, the development of more effective and easier problem-solving procedures and the expansion of this model's applications should be studied in the near future.

\section{Acknowledgments}

I would like to express my sincere thanks to Referees for their thoughtful advice and painstaking corrections. I would also like to thank Professor Shaw-Ping Lan for his guidance and Matthew Clarke for his English revisions and English lessons.

\section{References}

[1] G. Alexouda and K. Paparrizos: A genetic algorithm approach to the product line design problem using the seller's return criterion: An extensive comparative computational study. European Journal of Operational Research, 134-1 (2001) 165-178.

[2] W. Chung: Mode, size, and location of foreign direct investments and industry markups. Journal of Economic Behavior 6 Organization, 45 (2001) 185-211.

[3] K. Gielens and G. Dekimpe: Do international entry decisions of retail chains matter in the long run. International Journal of Research in Marketing, 18 (2001) 235-259.

[4] D. E. Goldberg: Genetic Algorithms in Search Optimization, and Machine Learning (Addison-Wesley, 1989).

[5] C. W. L. Hill, H. Peter and W. C. Kim: An eclectic theory of the choice of international entry mode. Strategic Management Journal, 11-2 (1990) 117-128.

[6] J. H. Holland: Adapation in Natural and Artificial Systems (University of Michigan Press, Ann Arbor, 1975). 
[7] K. Iwamura and L. Baoding: Dependent-chance integer programming applied to capital budgeting. Journal of the Operations Research Society of Japan, 42-2 (1999) 117-127.

[8] J. H. Jaramillo, J. Bhadury and R. Batta: On the use of genetic algorithms to solve location problems. Computers and Operations Research, 29-6 (2002) 761-779.

[9] T. Kangrong and T. Shozo: Optimization of fuzzy inference rules by using the genetic algorithm and its application to the bond rating. Journal of the Operations Research Society of Japan, 42-3 (1999) 302-315.

[10] C. F. Liaw: A hybrid genetic algorithm for the open shop scheduling problem. European Journal of Operational Research, 124-1 (2000) 28-42.

[11] H. E. Maraghy, V. Patel and I. B. Abdallah: Scheduling of manufacturing systems under dual-resource constraints using genetic algorithms. Journal of Manufacturing System, 19-3 (2000) 186-201.

[12] T. L. Saaty: The Analytic Hierarchy Process (McGraw-Hill, New York, 1980).

[13] M. Sakawa, K. Kato and S. Ushiro: Operational planning of district heating and cooling plants through genetic algorithms for mixed 0-1 linear programming. European Journal of Operational Research, 137-3 (2003) 677-687.

[14] Y. Yoshitomi, H. Ikenoue, T. Takeba and S. Tomita: Genetic algorithm in uncertain environments for solving stochastic programming problem. Journal of the Operations Research Society of Japan, 43-2 (2000) 266-290.

Cheng-Chang Chang

National Defense University

Chung-Ho, Taipei 235, Taiwan

Republic of China (ROC)

E-mail: coc26@rs590.ndmc.edu.tw 